\title{
Programa de la Reforma Educativa: Dimensión y análisis de sus lineamientos
}

\author{
Program of Educational Reform: Dimension \\ and analysis of its guidelines
}

\begin{abstract}
Adrián Frausto Martín del Campo*
\section{RESUMEN}

Se analiza un programa clave del sistema educativo nacional. El Programa de la Reforma Educativa cuenta con uno de los mayores presupuestos asignados por la actual administración federal. Este programa asigna recursos a las comunidades escolares para la mejora del rezago de la infraestructura de las escuelas, que están ubicadas en zonas de bajos recursos. A partir de los lineamientos de información de bases de datos publicadas en el portal de transparencia del programa, se analizan las similitudes y diferencias entre el Programa Escuelas de Excelencia para Abatir el Rezago Educativo y el programa en cuestión. Además se presenta un estudio cuantitativo de la cobertura y datos financieros, entre otros, del programa en el ciclo 2014-2015.

Palabras clave: Programa de la Reforma Educativa, política pública, programas educativos, Programa Escuelas de Excelencia para Abatir el Rezago Educativo

\section{ABSTRACT}

A key program of the national education system is analyzed: the Education Reform Program has one of the largest budgets allocated by the current federal administration. This program transfers resources to school communities to improve the infrastructure lag schools themselves that are located in low-income areas. From guidelines database information published on the website of transparency of the program, the similarities and differences are analyzed between the Excellence Schools to Reduce the Educational Backwardness Program and the program in question. In addition, a quantitative study of coverage and financial data is presented of the program in the cycle 2014-2015.

Keys words: Education Reform Program, education policy, education programs, the Excellence Schools to Reduce the Educational Backwardness Program
\end{abstract}

\section{INTRODUCCIÓN}

El Programa Escuelas de Excelencia para Abatir el Rezago Educativo (PEEARE) se convirtió en el programa bandera de la tan mencionada Reforma Educativa en México (PRE). Sin más, de un ciclo a otro, se mostró como la principal acción gubernamental del presente sexenio en materia de mejora de la educación para el alumnado de educación básica pero, ¿a cuánta población atiende

*Centro de Estudios Educativos; adrian.frausto@cee.edu.mx 
realmente este programa? ¿Es de carácter universal? ¿Es un programa integral que entiende que los problemas de aprendizaje son multifactoriales?

A continuación se presenta un análisis del PEEARE y del PRE en dos partes. La primera compara los lineamientos de operación del PEEARE (el de junio y el de diciembre de 2014) y el acuerdo correspondiente al documento que emite los propios del PRE; el propósito es mostrar la estructura que se mantuvo en los acuerdos y las diferencias. La segunda parte es un análisis sobre la cobertura del PEEARE. ${ }^{1}$

\section{PARALELISMOS Y DIFERENCIAS ENTRE PEEARE Y PRE}

En junio de 2014, a poco más de un año que el ejecutivo y el entonces secretario de Educación Pública, Emilio Chuayffet Chemor, promulgaran la reforma educativa, se publicó en el Diario Oficial de la Federación (DOF) el acuerdo 05/06/14 por el que se emitieron Los lineamientos de operación del Programa Escuelas de Excelencia para Abatir el Rezago Educativo. La operación y ejecución del programa requirió cambios en los documentos normativos, por lo que, en diciembre del mismo año, el DOF publicó el acuerdo número 28/12/14 por el que se modificaron dichos lineamientos (en adelante PEEARE-Modificado).

Nueve meses después, ahora bajo la tutela del secretario Aurelio Nuño Mayer, la SEP publicó a través del DOF los Lineamientos de operación del Programa de la Reforma Educativa bajo el acuerdo $11 / 09 / 15$, que dejaron sin vigor los acuerdos anteriores, correspondientes al PEEARE. No se llevó a cabo un cambio sustancial, siendo lo más significativo la modificación al nombre y la inclusión de un componente específico para la instalación y mantenimiento de bebederos, en detrimento de estrategias educativas para abatir el rezago educativo, bajo la premisa de que "el PEEARE fue identificado con la Reforma Educativa, por 'responder' a los mandatos de tal reforma" (SEP, 2015).

Lo que se mantiene en los tres acuerdos:

\footnotetext{
${ }^{1}$ Hasta el momento el PRE está en ejecución por lo que aún no hay cifras cerradas.
} 
1. La estructura de los contenidos del índice es la misma en los tres acuerdos. Los temas principales son: 1. Objetivos, 2. Lineamientos generales, 3. Responsabilidades, 4. Operación del programa, 5. Instancias, 6. Auditoría, control y seguimiento, 7. Transparencia y rendición de cuentas, y 8. Quejas y denuncias. No obstante, en el PRE se incluyeron dos incisos que serán descritos más adelante, en el numeral 2 de la siguiente sección.

2. El objetivo general planteado en el primer acuerdo del PEEARE no sufrió cambios en el acuerdo de las modificaciones ni en el del PRE, a saber: "Contribuir a la disminución del rezago en las condiciones físicas de las escuelas públicas de educación básica y al fortalecimiento de la autonomía de gestión para mejorar la prestación de servicios con calidad y equidad".

3. Los cuatro objetivos específicos del acuerdo del PEEARE y PEEARE-Modificado son los mismos: el primero hace referencia a la mejora de la infraestructura y equipamiento de las escuelas, el segundo al fortalecimiento de la autonomía escolar, el tercero a las estrategias para inhibir los factores escolares asociados al rezago educativo, y el cuarto al apoyo a la supervisión de zona. En el documento del PRE se mantuvieron los dos primeros objetivos específicos, pero se modificaron los dos últimos, como se mostrará más adelante.

4. La cobertura de los programas se mantuvo en las 32 entidades federativas de país.

Principales diferencias entre los tres acuerdos:

1. El PEEARE planteó como población objetivo del programa a las escuelas públicas de educación básica. Sin embargo, el PEEARE-Modificado especificó que las comunidades escolares de las escuelas públicas beneficiadas serían la población objetivo, y definió comunidad escolar como el colectivo conformado entre el Consejo Escolar de Participación Social y el Director de las escuelas participantes; por tanto, según el transitorio segundo, las acciones deberían ser desarrolladas de manera mancomunada entre estos dos actores. Los lineamientos del PRE mantuvieron a lo largo del documento el sentido de comunidad escolares. 
2. Se modificó el PEEARE para establecer que la SEP emitiría el Esquema simplificado para el ejercicio y comprobación de recursos referente al inciso l) del numeral 3.1 de los lineamientos. En el PRE se menciona nuevamente pero en el inciso h) del mismo numeral, especificando para el caso de los componentes 1 y 2, y que sería la SEP, a través de la Dirección General de Desarrollo de la Gestión e Innovación Educativa (DGDGIE) quien asumiría las responsabilidades.

3. A diferencia del PEEARE y del PEEARE-Modificado, que en cuyos lineamientos no se menciona el presupuesto, ${ }^{2}$ el PRE señaló el monto del recurso asignado por el ramo 11 de Educación pública (con un total de \$9067248270.00) como parte del anexo 18, "Recursos para la atención de niñas, niños y adolescentes" del Presupuesto de Egresos de la Federación para el ejercicio fiscal 2015. Además, señala que 15\% de dicho recurso deberá utilizarse en la instalación y mantenimiento de bebederos de agua potable para consumo humano.

4. Respecto a los objetivos específicos, dos son diferentes. El objetivo del PEEARE en torno a la implementación de estrategias orientadas para inhibir los factores escolares asociados a la producción de rezago educativo en las escuelas públicas de básica, fue sustituido por la instalación y mantenimiento de bebederos escolares. El segundo objetivo modificado del PEEARE al PRE fue la reformulación del apoyo a la supervisión escolar en materia de su fortalecimiento para un mejor acompańamiento a las escuelas.

5. En cuanto a los requisitos para la participación del programa por parte de las escuelas o comunidades escolares, en el PEEARE y PEEARE-Modificado son los mismos, a saber, entregar una carta compromiso a la Autoridad Educativa Local (AEL) donde se establecerá el compromiso de recibir asistencia técnica por parte de las autoridades. En el PRE se detalla el procedimiento, donde se indica que la carta compromiso se entregará a la supervisión de zona, y en el caso de la Comi-

\footnotetext{
${ }^{2}$ Aunque en el presupuesto de egresos de la federación para el ejercicio fiscal 2014 el monto asignado para el PEEARE fue de \$7567248270.00 (Siete mil quinientos sesenta y siete millones doscientos cuarenta y ocho mil doscientos setenta pesos 00/100 M.N.).
} 
sión Nacional de Fomento Educativo (CONAFE), a la Acción Promotora de Educación Comunitaria (APEC). También se pide integrar el acta de planeación del ejercicio de los recursos alineado a la Ruta de Mejora.

6. En el PRE se establece de manera inédita el numeral 2.5.2 sobre la responsabilidad de la supervisión escolar, la cual debe entregar una carta compromiso a las AEL en su decisión de participar en el Programa. El lenguaje no es claro y puede interpretarse que la o el supervisor no quiera participar en el programa, aunque las escuelas de su zona participen en él. Esta ambigüedad podría dar pie a problemáticas serias para la implementación y, sobre todo, para la operación del programa.

7. Los montos asignados se muestran en el cuadro 1. Se presenta la comparación según tipo de escuela, componente y ciclo escolar. Para el ciclo 2015-2016 los lineamientos presentaron dos tabuladores: uno para escuelas de nueva incorporación y otro para escuelas de habían participado en el ciclo 2014-2015. Se observa un cambio en el recurso destinado a la supervisión, además de que en el PRE se designa como componente 3, en el PEEARE cada entidad podía recibir hasta cinco millones de pesos, en tanto que en el PRE se establece que cada entidad podrá recibir hasta $2 \%$ del monto asignado del programa. En el tabulador del PRE se observa una disminución en los montos que son de nueva incorporación, comparados con los del ciclo anterior, mientras que las escuelas de reincorporación reciben, en algunos casos, menos de la mitad de lo que recibieron en el ciclo 2014-2015. 


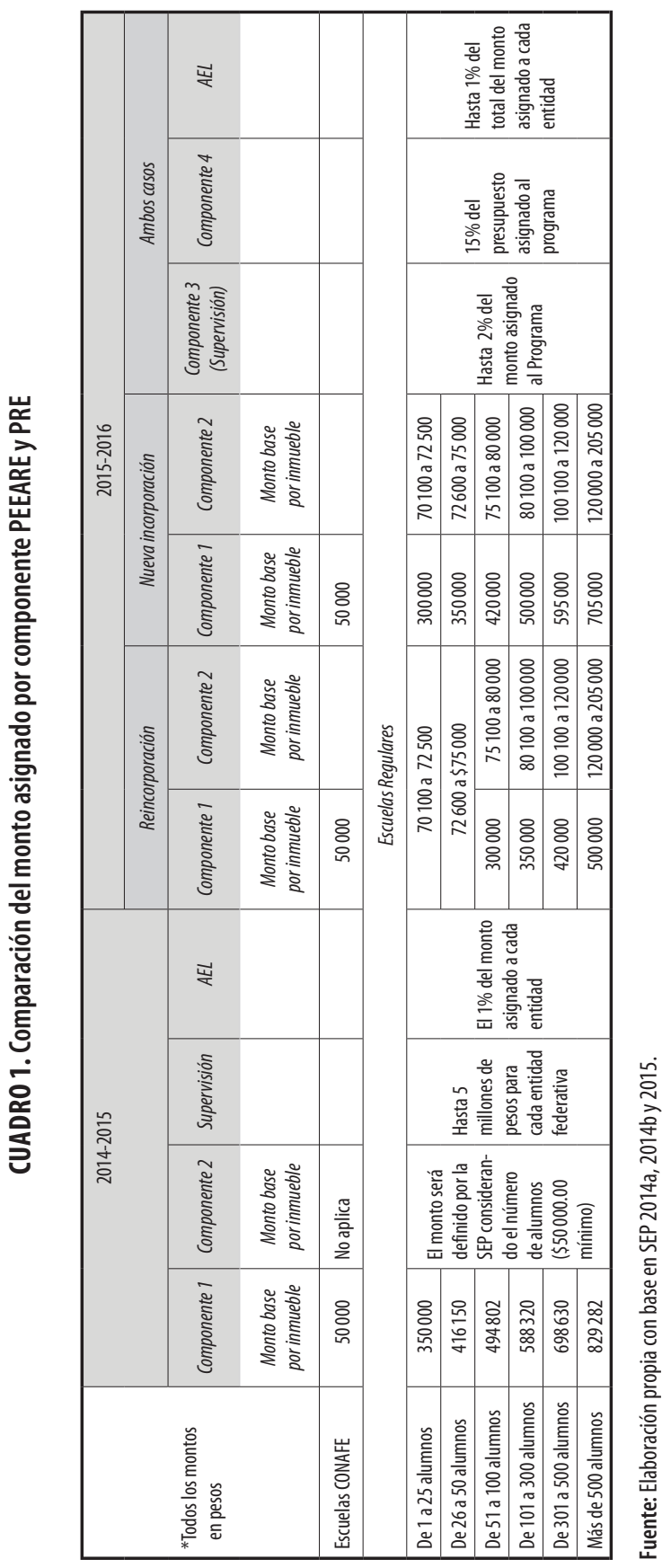




\section{ANÁLISIS DESCRIPTIVO DEL PEEARE 2014-2015}

En portal de transparencia presupuestaria de la Reforma Educativa se publicaron los datos abiertos de PEEARE para el ciclo 2014-2015. A continuación se presenta un análisis comparativo de una selección de elementos que permiten dimensionar la cobertura, el tipo y modalidad de escuelas que atendió, los recursos económicos asignados por componente y el monto ejercido, así como las acciones llevadas a cabo para cumplir los objetivos, según los dos componentes manifestados en los lineamentos del acuerdo 05/06/14 y de su modificación en el acuerdo 28/12/14. Con base en la información brindada por el portal en el archivo "Diccionario de datos", la fecha de corte de la información fue entre septiembre y octubre de 2015.

La gráfica 1 presenta la comparación entre el número de escuelas del sistema educativo nacional (SEN), según cifras de la SEP, y el número nacional de escuelas que pertenecían al padrón de ciclo escolar 2014-2015 de comunidades educativas beneficiarias del Programa, como parte del Censo de Escuelas Maestros y Alumnos de Educación Básica y Especial (CEMABE) (PEEARE CEMABE), y el número nacional de escuelas que fueron validadas por la Autoridad Educativa Local (PEEARE VALIDADA AEL), las cuales recibieron el recurso. Del total de escuelas de educación básica que tiene el SEN solamente el 10\% recibió apoyo por el programa. De las escuelas de preescolar el 3.24\%, las de primaria el $14.57 \%$ y las de nivel secundaria tan solo el $13.23 \%$. 
REVISTA LATINOAMERICANA DE ESTUDIOS EDUCATIVOS, VOL. XLVI, NÚM. 1, 2016

\section{GRÁFICA 1. Escuelas del PEEARE, 2014-2015}

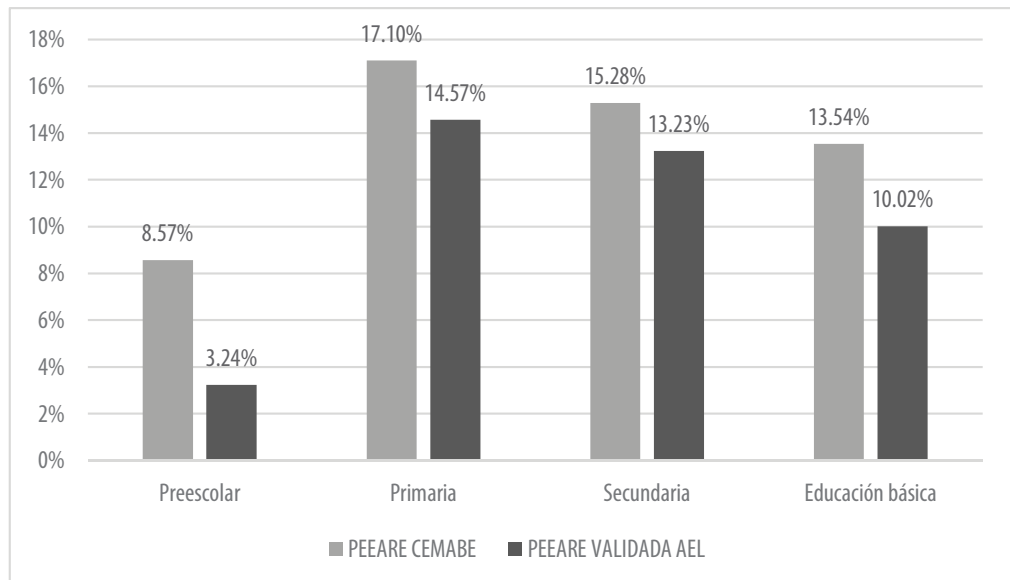

Fuente: Elaboración propia con base en: SCHP-SEP, 2015.

El total de las escuelas validadas por las AEL fue de 20135 , lo que representa $74 \%$ de las escuelas beneficiadas que estaban en el padrón del CEMABE con alto y muy alto rezago en infraestructura, obtenido a partir del Índice de Carencia por Escuela (ICE) elaborado por la SEP. Del total de escuelas validadas y beneficiadas las de tipo regular fueron de mayor número que las CONAFE, con 15159 y 4976 escuelas, respectivamente.

\section{GRÁFICA 2. Escuelas del PEEARE según tipo, 2014-2015}

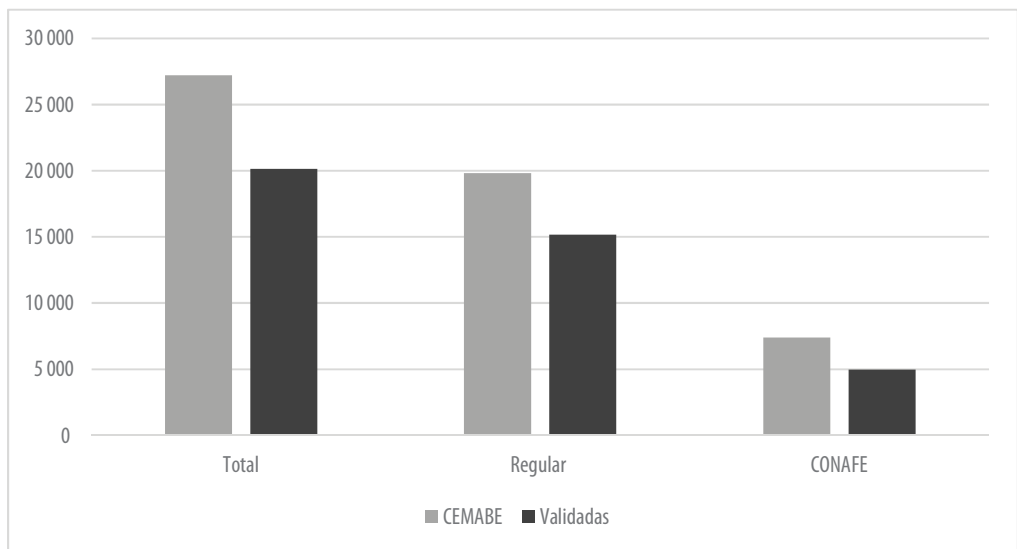

Fuente: Elaboración propia con base en: SCHP-SEP, 2015. 
El comparativo del número de escuelas validadas por las autoridades educativas estatales, respecto del total nacional de las escuelas en su modalidad para el ciclo 2014-2015, arrojó que a CONAFE, la mayor tasa, $15.04 \%$, le siguió de cerca telesecundaria con $14.24 \%$ e indígena con $13.89 \%$. Primaria general y secundarias técnicas tienen tasas menores a $8 \%$.

CUADRO 2. Escuelas del PEEARE según modalidad, 2014-2015

\begin{tabular}{|l|c|c|c|c|}
\hline \multicolumn{1}{|c|}{ MODALIDAD } & $\begin{array}{c}\text { TOTAL } \\
\text { SEN }\end{array}$ & PEEARE CEMABE & $\begin{array}{c}\text { PEEARE VALIDADA } \\
\text { AEL }\end{array}$ & $\begin{array}{c}\text { Participación nacional } \\
\text { escuelas validadas }\end{array}$ \\
\hline CONAFE & 34503 & 7401 & 5188 & $15.04 \%$ \\
\hline General & 117779 & 11657 & 9207 & $7.82 \%$ \\
\hline Indígena & 19751 & 4918 & 2743 & $13.89 \%$ \\
\hline Secundaria técnica & 4709 & 366 & 335 & $7.11 \%$ \\
\hline Telesecundaria & 18700 & 2872 & 2662 & $14.24 \%$ \\
\hline
\end{tabular}

Fuente: Elaboración propia con base en: SCHP-SEP, 2015.

Respecto a la distribución de las escuelas por nivel, en primaria fue donde se reportó el más alto porcentaje de las que recibieron recursos para cumplir con los objetivos del programa, 65\%; en tanto que en secundaria fueron solo $22 \%$ las que estaban en esa condición, y en preescolar $12 \%$.

GRÁFICA 3. Escuelas del PEEARE validadas por la AEL por nivel educativo, 2014-2015

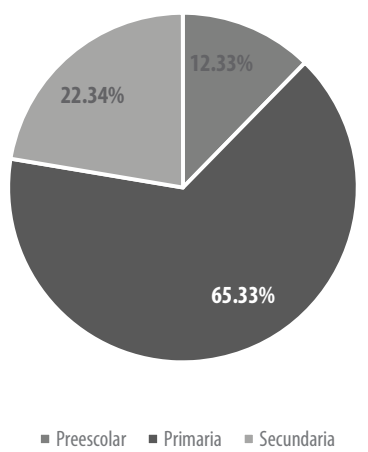

Fuente: Elaboración propia con base en: SCHP-SEP, 2015. 
Del total de las escuelas validadas y que recibieron el recurso para la ejecución del proyecto, casi la mitad corresponden a la escuelas generales, 25\% a CONAFE, el resto a las otras modalidades. Si restamos el porcentaje de secundarias de modalidad técnica y tele del porcentaje de secundarias de la gráfica anterior, el resultado es $7.46 \%$ de secundarias de modalidad general; esto indica que el número de telesecundarias fue mayor respecto a las otras modalidades de este mismo nivel educativo.

GRÁFICA 4. Escuelas del PEEARE validadas por la AEL por modalidad, 2014-2015

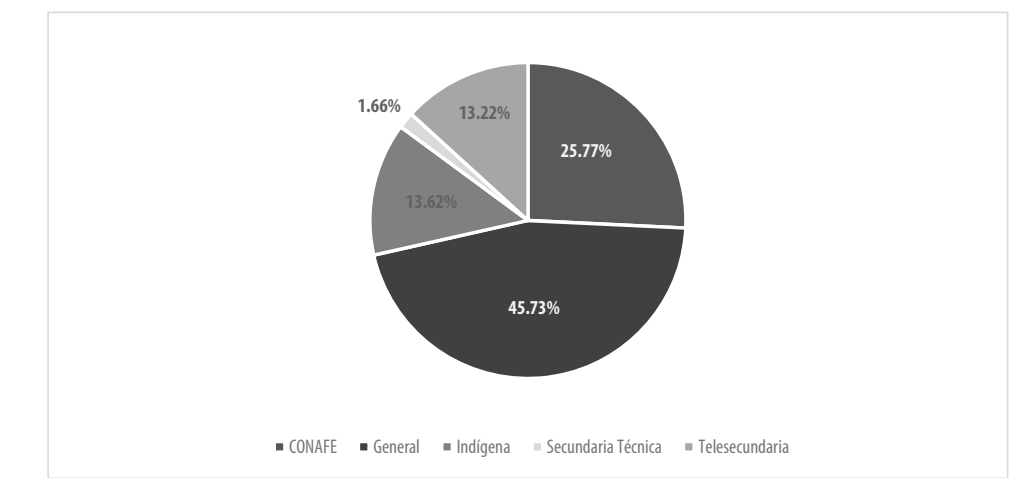

Fuente: Elaboración propia con base en: SCHP-SEP, 2015.

La base de datos define el grado de rezago en dos niveles, alto y muy alto. Según los lineamientos del PEEARE y PEEARE-Modificado, la población objetivo son las comunidades educativas de las "escuelas públicas de educación básica de mayor rezago en sus condiciones físicas y de equipamiento. Las entidades con mayor número de escuelas validadas con alto grado de rezago son: Veracruz (2728), Guerrero (1 821), Chiapas (1 180), San Luis Potosí (977) y Oaxaca (881). Los estados con mayor número de escuelas validadas con muy alto grado de rezago son: Chiapas (984), Guerrero (837), Veracruz (707), Oaxaca (388) y Durango (244). 
GRÁFICA 5. Escuelas del PEEARE validadas por la AEL según grado de rezago en infraestructura, 2014-2015

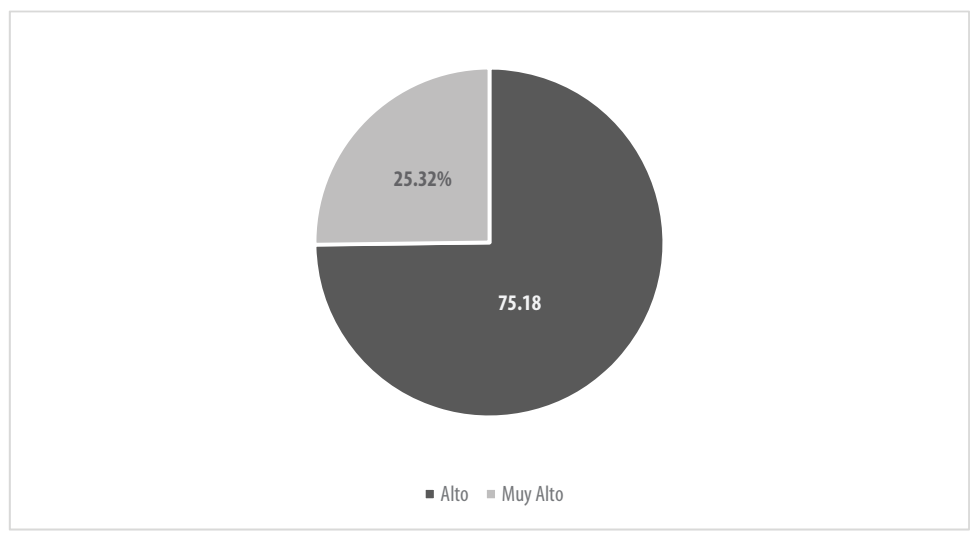

Fuente: Elaboración propia con base en: SCHP-SEP, 2015.

El programa divide el apoyo financiero dirigido a las comunidades escolares en dos componentes. El primero estaba enfocado a la construcción, rehabilitación y compra de mobiliario. Según la base de datos del portal de transparencia presupuestaria de la Reforma Educativa, la acción de construcción fue la más ejecutada: $60 \%$ de las escuelas reportó haber realizado alguna acción de construcción, mantenimiento o rehabilitación de algún espacio educativo. 58\% de las escuelas realizaron acciones en infraestructura hidrosanitaria, y casi 50\% compró mobiliario.

El componente 2 comprendía acciones para fortalecer la gestión escolar e implementar acciones para contribuir a la Normalidad mínima ${ }^{3}$ de operación escolar. De acuerdo con lo reportado por las escuelas, casi la mitad de ellas realizó alguna acción en este componente; 36\% realizó acciones para contribuir a la mejora de las competencias de lectura, escritura y matemáticas; una cuarta parte las llevó a cabo para contribuir a la prevención del rezago y abandono escolar. Y menos de $10 \%$ las destinó a contribuir al desarrollo de la convivencia escolar.

En la gráfica 6 se incluyen otros datos como el porcentaje de escuelas que pertenecen a alguno de los municipios participantes

\footnotetext{
${ }^{3}$ Se define como el conjunto de las acciones básicas indispensables de primero orden, que deben cumplirse en cada escuela para el buen desempeño de la tarea docente y el logro de aprendizaje del alumnado.
} 
en la Cruzada Nacional Contra el Hambre (en el caso del ciclo 2014-2015, representan 55.8\% de las escuelas beneficiadas); y las que, además, participaron en el mismo ciclo en el Programa de Escuelas de Tiempo Completo (11.2\%).

GRÁFICA 6. Acciones por componente en escuelas del PEEARE validadas por la AEL, 2014-2015

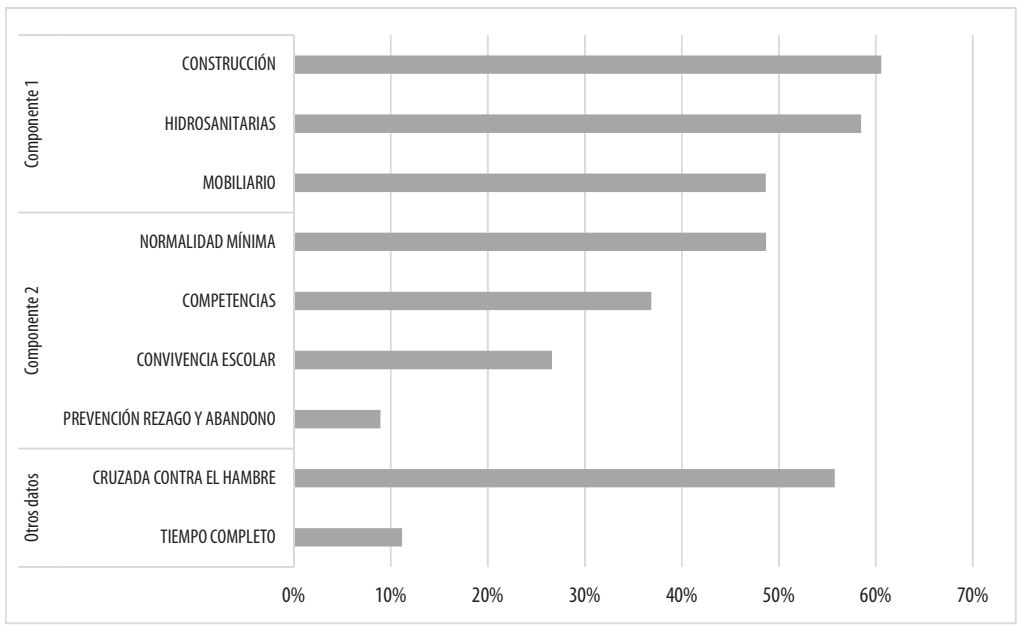

Fuente: Elaboración propia con base en: SCHP-SEP, 2015.

En el gráfico 7 se muestra la concentración de número de escuelas por región. Aunque la implementación del PEEARE es previa a la regionalización que emprendió el secretario Aurelio Nuño al principio de su administración, esta división geográfica ayuda a visualizar hacia dónde se enfocó la atención del programa. La región Sur-Sureste fue la que contó con mayor número de escuelas, esta región comprende los estados de mayor grado de marginación y menor índice de desarrollo humano del país: Campeche, Chiapas, Guerrero, Oaxaca, Quintana Roo, Tabasco, Veracruz y Yucatán, con un total de 10748 escuelas.

La región que le sigue en número de escuelas beneficiadas es la Occidente, y la conforman: Aguascalientes, Colima, Guanajuato, Jalisco, Michoacán, Nayarit, Querétaro y Zacatecas. Después está la región Noroeste, con 2248 escuelas beneficiadas, cuyos estados son: Coahuila, Durango, Nuevo León, San Luis Potosí 
y Tamaulipas. La región del Centro es la penúltima en número de escuelas atendidas, con 2248, los estados son: Distrito Federal, Estado de México Hidalgo, Morelos, Puebla y Tlaxcala. Por último, la región con menor número de escuelas en el programa fue la Noreste, cuyos estados son: Baja California, Baja California Sur, Chihuahua, Sinaloa y Sonora.

GRÁFICA 7. Distribución regional de escuelas del PEEARE validadas por la AEL, 2014-2015

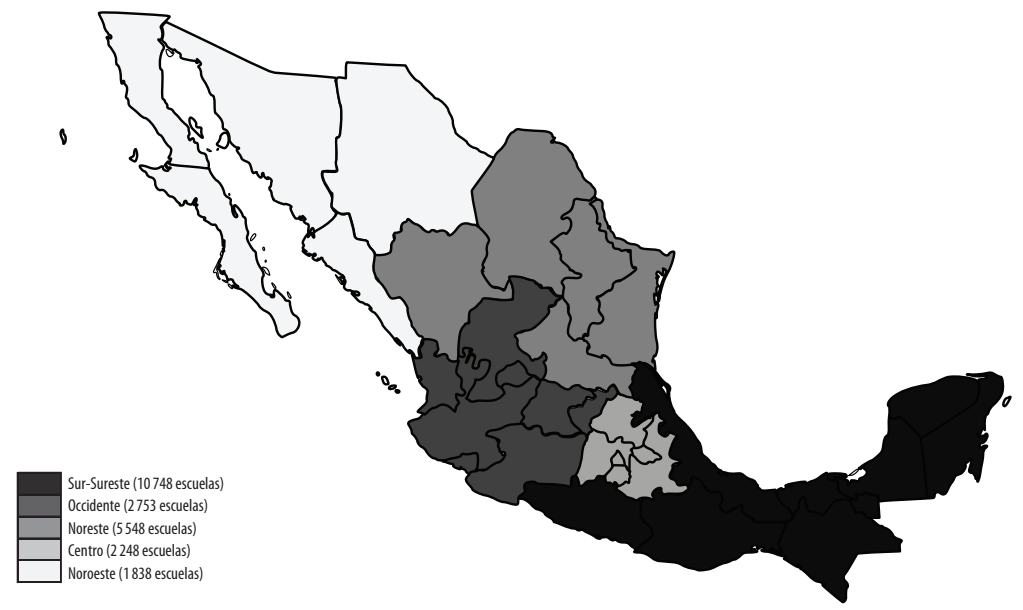

Fuente: Elaboración propia con base en: SCHP-SEP, 2015.

En cuanto a la información financiera del PEEARE, las escuelas de tipo regular del Componente 2 representan solo $20 \%$ del monto destinado al Componente 1 . Por su parte, los lineamientos de operación establecieron que CONAFE no recibiría apoyo económico para el Componente 2 (sin dar razón de ello), el cual, comparado con el monto asignado en este mismo componente a las escuelas de tipo regular, representa solo $4 \%$. Del total del recurso ejercido, las escuelas de tipo regular concentraron $97 \%$ contra 3\% de las escuelas comunitarias. Esto indica que el recurso del programa se encauzó prácticamente para atender obras de construcción, rehabilitación, mantenimiento y adquisición de 
mobiliario escolar, priorizando a las escuelas regulares. La gráfica 8 muestra la distribución de los componentes en los dos tipos de escuelas.

CUADRO 3. Total del monto* asignado por componente y total ejercido en escuelas PEEARE validadas por la AEL, 2014-2015

\begin{tabular}{|l|r|r|r|}
\hline \multicolumn{1}{|c|}{ Tipo de escuela } & Componente 1 & Componente 2 & Total Ejercido \\
\hline Regular & 5703299423.24 & 1253993788.33 & 6835222863.80 \\
\hline CONAFE & 243150000.00 & 0.00 & 239066380.26 \\
\hline Total & 5946449423.24 & 1253993788.33 & 7074289244.06 \\
\hline
\end{tabular}

Fuente: Elaboración propia con base en: SCHP-SEP, 2015. * Precios en pesos

GRÁFICA 8. Distribución del monto asignado por componente y tipo de escuela, 2014-2015

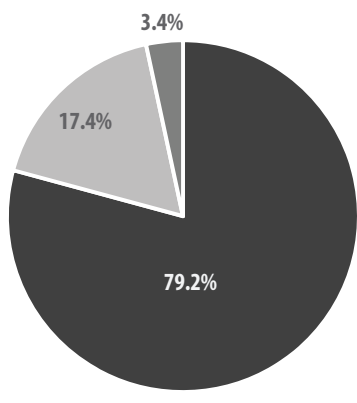

- Componente 1 Regular In Componente 2 Regular - Componente 1 CONAFE

Fuente: Elaboración propia con base en: SCHP-SEP, 2015.

\section{PARA DIALOGAR}

Tanto el contenido de los acuerdos como de los lineamientos aluden a los propósitos educativos de los programas; sin embargo, el PEEARE y el PRE están estructurados para destinar recursos a la mejora de la infraestructura. La infraestructura es condición necesaria, pero no suficiente para garantizar la mejora educativa 
en su más amplia expresión. Esta no se agota en las condiciones físicas de las escuelas; para lograrla, se precisa considerar, entre otros, los contenidos de los programas, los materiales educativos, la formación docente y del personal directivo.

Se establece en los lineamientos de operación del PEEARE y PRE que el personal directivo de las escuelas y los Consejos de Participación Social serán los ejecutores de los programas, ¿en verdad ambos actores están preparados técnicamente para administrar, ejecutar y rendir cuenta de los recursos en función de objetivos específicos? Es verdad que los lineamientos explicitan que estas figuras recibirán acompañamiento por parte de la supervisión de zona, ¿este actor estará preparado a su vez para orientar en temas como el diseño arquitectónico y estructural de una construcción o rehabilitación de una escuela?

Aunado a lo anterior, no está en el horizonte de los lineamientos la diferencia entre las escuelas de organización multigrado respecto a las de organización completa. El hecho de que un número de maestros, menor al número de grupos participen en el programa, y uno de ellos tenga las funciones de la dirección, hace que el programa abone carga administrativa a las ya de por sí numerosas funciones que debe atender una directora o un director.

Es entendible que el programa no puede atender la totalidad de las escuelas de la base de datos del CEMABE. La disponibilidad presupuestal de cada año fiscal está sujeta a variables que salen del control de la SEP; sin embargo, el programa no está enmarcado en un plan de gradualidad de atención; es decir, en priorizar las escuelas con muy alto grado de rezago en infraestructura, de modo que, una vez atendidas estas, se siguiera con las de alto rezago.

El dato del número de escuelas que recibieron recursos para el Componente 1 indica la prioridad del programa: la construcción. PEEARE y PRE son acciones gubernamentales que atienden una necesidad puntual de las escuelas públicas de nivel básico: la falta de espacios físicos adecuados y el deterioro de los mismos por el uso y paso del tiempo. Bajo la premisa de que el Estado es el que otorga un monto y las comunidades escolares "deciden" el destino del recurso, ¿̇será posible pensar en un modelo de intervención que quede instalado, de modo que la organización entre directivos y familiares sea permanente para atender asuntos referentes 
de la escuela independientemente de la asignación de recursos por parte del gobierno?

Por último, se presentan dos tipos de diferencias en el análisis comparativo entre el PEEARE y el PRE, unas de forma y otras de fondo. Respecto a las primeras se observó una reestructuración del modo de presentar la asignación de los montos; el PRE divide en componentes que indican los recursos ad intra y ad extra de la comunidad educativa. En cuanto a una importante diferencia de fondo, tenemos la sustitución del objetivo (en el PEEARE) de atención a la implementación de estrategias orientadas a inhibir los factores escolares asociados a la producción de rezago educativo, por la implementación (en el PRE) de la construcción y mantenimiento de bebederos.

\section{REFERENCIAS BIBLIOGRÁFICAS}

Secretaría de Educación Pública (SEP). 2014a. "Acuerdo número 05/06/14 por el que se emiten los lineamientos de operación del Programa Escuelas de Excelencia para Abatir el Rezago Educativo", México. Disponible en http://dof.gob.mx/nota_detalle.php? codigo $=534915$ 6\&fecha=18/06/2014. Fecha de acceso, marzo de 2016.

Secretaría de Educación Pública (SEP). 2014b. "Acuerdo número 28/12/14 por el que se modifican Los Lineamientos de Operación del Programa Escuelas de Excelencia para Abatir el Rezago Educativo", México. Disponible en http:// www.dof.gob.mx/nota_detalle.php?codigo $=5377405 \& \mathrm{fec}$ ha=26/12/2014. Fecha de acceso, marzo de 2016.

Secretaría de Educación Pública (SEP). 2015. “Acuerdo número 11/09/15 por el que se emiten los Lineamientos de Operación del Programa de la Reforma Educativa”, México. Disponible en http://dof.gob.mx/nota_detalle.php?codigo=54 08422\&fecha=15/09/2015. Fecha de acceso, marzo de 2016.

Secretaría de Hacienda y Crédito Pública-Secretaría de Educación Pública (SHCP-SEP), 2015. "Transparencia Presupuestaria. Programa de la Reforma Educativa”. Disponible en http://escuelas.transparenciapresupuestaria.gob.mx/\# Fecha de acceso, febrero de 2016. 